\title{
Article
}

\section{Proteomics as a Complementary Tool for Identifying Unintended Side Effects Occurring in Transgenic Maize Seeds As a Result of Genetic Modifications}

Lello Zolla, Sara Rinalducci, Paolo Antonioli, and Pier Giorgio Righetti

J. Proteome Res., 2008, 7 (5), 1850-1861• DOI: 10.1021/pr0705082 • Publication Date (Web): 05 April 2008

Downloaded from http://pubs.acs.org on March 2, 2009

\section{More About This Article}

Additional resources and features associated with this article are available within the HTML version:

- $\quad$ Supporting Information

- $\quad$ Links to the 1 articles that cite this article, as of the time of this article download

- Access to high resolution figures

- $\quad$ Links to articles and content related to this article

- $\quad$ Copyright permission to reproduce figures and/or text from this article

\section{View the Full Text HTML}




\title{
Proteomics as a Complementary Tool for Identifying Unintended Side Effects Occurring in Transgenic Maize Seeds As a Result of Genetic Modifications
}

\author{
Lello Zolla, ${ }^{,+\ddagger}$ Sara Rinalducci, ${ }^{\ddagger}$ Paolo Antonioli, ${ }^{\circledR}$ and Pier Giorgio Righetti ${ }^{\circledR}$ \\ Department of Environmental Sciences, University of Tuscia, Viterbo, Italy, and Department of Chemistry, \\ Materials and Engineering Chemistry "Giulio Natta", Polytechnic of Milan, Milan, Italy
}

Received August 6, 2007

\begin{abstract}
To improve the probability of detecting unintended side effects during maize gene manipulations by bombardment, proteomics was used as an analytical tool complementary to the existing safety assessment techniques. Since seed proteome is highly dynamic, depending on the species variability and environmental influence, we analyzed the proteomic profiles of one transgenic maize variety (event MON 810) in two subsequent generations (T05 and T06) with their respective isogenic controls (WT05 and WT06). Thus, by comparing the proteomic profiles of WT05 with WT06 we could determine the environmental effects, while the comparison between WT06 and T06 seeds from plants grown under controlled conditions enabled us to investigate the effects of DNA manipulation. Finally, by comparison of T05 with T06 seed proteomes, it was possible to get some indications about similarities and differences between the adaptations of transgenic and isogenic plants to the same strictly controlled growth environment. Approximately 100 total proteins resulted differentially modulated in the expression level as a consequence of the environmental influence (WT06 vs WT05), whereas 43 proteins resulted up- or down-regulated in transgenic seeds with respect to their controls (T06 vs WT06), which could be specifically related to the insertion of a single gene into a maize genome by particle bombardment. Transgenic seeds responded differentially to the same environment as compared to their respective isogenic controls, as a result of the genome rearrangement derived from gene insertion. To conclude, an exhaustive differential proteomic analysis allows to determine similarities and differences between traditional food and new products (substantial equivalence), and a case-by-case assessment of the new food should be carried out in order to have a wide knowledge of its features.
\end{abstract}

Keywords: transgenic seeds • maize • event MON $810 \bullet$ proteomics $\bullet$ tandem mass spectrometry

\section{Introduction}

The introduction of exogenous DNA sequences into the plant genome for GMO production is commercially carried out by either Agrobacterium-mediated transformation (i.e., infecting plant cells with a disarmed pathogenic organism like Agrobacterium tumefaciens containing the transgene) or particle bombardment (i.e., bombardment of cells with metal particles carrying the transgene). In both cases, the process is random, leading to physical disruption in the genome (e.g., insertional mutation) or gene regulation (pleiotropic effect) and possible inactivation of endogenous genes. ${ }^{1}$ Activation of silent genes and formation of fusion proteins by transcriptional readthrough processes are also possible, ${ }^{2}$ although these may be in part minimized during the process of transgenic product development. ${ }^{3}$ In addition, the transcriptional products of the introduced gene(s) (and possibly the modified biochemical

* Corresponding author: Prof. Dr. Lello Zolla, University of Tuscia, Largo dell'Università snc, 01100 Viterbo, Italy. Phone: 00390761 357100. Fax: 0039 0761 357179. E-mail: zolla@unitus.it.

‡ University of Tuscia.

$\S$ Polytechnic of Milan. pathway based on them) may interact with the regulation of other genes or biochemical pathways. A major concern is the possible occurrence of unintended effects caused, for example, by the site of transgene integration (e.g., interruption of important open reading frames or regulatory sequences), which could result in modified metabolism, novel fusion proteins, or other pleiotropic effects that could compromise the product safety. ${ }^{4,5}$ This includes the production of new allergens or toxins. Thus, although people has been consuming genetically modified (GM) crops for many years, there is interest in assessing their safety with the aim of addressing both the intended and the unintended toxic or nutritionally harmful side effects resulting from the cultivation and consumption of products from GM crops. ${ }^{6,7}$

The comparison between GM and non-GM crops usually comprises agronomic/phenotypic features, feed performance studies and crop composition. Molecular profiling may facilitate a more complete, holistic comparative analysis. This approach involves several technologies, like DNA microarrays, proteomics, mRNA profiling and metabolomic profiling, which are now available as complementary tools for the safety assessment of 
GM crops. ${ }^{4,8}$ The combination of these nontargeted approaches is considered to facilitate a more comprehensive approach than the targeted methods and, thus, to provide additional opportunities for identifying unintended effects.

The proteome is the entire complement of a genome and the result of genetic expression, ribosomal synthesis, and proteolytic degradation. ${ }^{9}$ Proteins are of special interest for the safety assessment because they could be toxins (e.g., phytohemagglutinin), antinutrients (e.g., protease inhibitors) and allergens, or they may be involved in their synthesis. Moreover, contrary to the genome, which is constant for an organism, the proteome is highly dynamic and depends on cell cycle, environmental influences, and tissue/cell type. Nevertheless it is the direct product of genome transcription and translation; therefore, if a genetic modification affects the genome of a plant by changing its metabolic pathways or producing new proteins, the resulting proteome will be altered. Thus, side by side to genomic, trascriptomic and metabolomic analysis of GM crops, a proteomic investigation represents a valuable analytical tool complementary to the existing safety assessment techniques, ${ }^{10}$ improving the probability of detecting unintended side effects.

The present study addresses the feasibility of proteomics in identifying unintended or intended changes in commercial GM maize caused by genetic engineering by comparing the transgenic seed proteome with the wild-type line. Moreover, since it is well-known that gene expression, and consequently the seed proteome, may be influenced by environmental conditions and variety, we have discriminated the recombinant DNA effects from those related to the environment by crosscomparisons between two consecutive generations of one stabilized transgenic maize line with their isogenic controls (WT06 vs WT05; T06 vs T05; T05 vs WT05; T06 vs WT06).

So far, two-dimensional gel electrophoresis (2DE) combined with mass spectrometry (MS) is still the most widely used approach for comparing plant proteomes in the attempt to identify differentially expressed proteins; ${ }^{11}$ thus, it was used in the present study. Our aim was to achieve a better understanding of the side effects generated by a single gene insertion (in this case, the sequence coding for the endotoxin CryIA(b) of Bacillus thuringiensis, Bt) on the expression profile of all the grain proteins, that is, to verify whether also nonspecific alterations of the maize genome can occur upon transformation. The Bt toxin is a protein toxic to insect pests, produced by the soil bacterium $B$. thuringiensis, which is transferred to plants to confer the ability to produce the insect toxin by its own. Some Bt maize varieties produce Bt toxin mainly inside the stalk, some mostly in the roots, while others express it in all parts of the plant. Thus, the GM maize seed proteome is of particular interest, because it is an important source for nutrients and, therefore, an essential part of the human diet.

\section{Material and Methods}

Chemicals and Samples. All the chemicals were purchased from Sigma-Aldrich. Acrylamide/Bis solution (40\%, 37.5:1), the Versa Doc Imaging System (model 3000) and the 17-cm long nonlinear immobilized $\mathrm{pH}$ gradients (IPG) 3-10 were obtained from Bio-Rad Laboratories (Hercules, CA). Glycine, sodium dodecyl sulfate (SDS), urea, thiourea, Tris(2-carboxyethyl) phosphine (TCEP), 2-hydroxyethyl disulfide and CHAPS were from Fluka. Bromophenol blue, carrier ampholytes, and agarose were purchased from Pharmacia Biotech (Uppsala, Sweden). HPLC grade acetonitrile (ACN) (Merck), Milli-Q water (Milli- pore, Bedford, MA), and formic acid (FA) (Sigma, St. Louis, MO) were used for the preparation of mobile phases.

Seed Collection and Plant Growth. The samples of maize seed used in this study were derived from the transgenic commercial Bt hybrid line 33P67 (event MON810 from Pioneer Hi-Bred International, Inc., Johnston, IA) and its respective control isoline 33P66. For our investigation, two subsequent generations of both lines were employed (i.e., WT05, WT06, where WT stands for "wild-type" and T05, T06 where T indicates "transgenic”). Commercial seeds (WT05, T05) were provided by Pioneer-Hi Bred Spain SL in 2005, whereas plants derived from the WT05 and T05 seeds were grown in Italy in 2006 side-by-side in environmentally controlled growth chambers, adjacent but separated, in order to obtain WT06 and T06 grains influenced by the same environmental effects. Plants were cultivated at $25{ }^{\circ} \mathrm{C}$ and $70 \%$ relative humidity with $14 \mathrm{~h}$ of light (50-100 $\left.\mu \mathrm{mol} \mathrm{m} \mathrm{m}^{-2} \mathrm{~s}^{-1}\right)$. It is important to note that the stability of the genome is guaranteed by the producer Company through multiple generations of crossing; therefore, the two subsequent generations here used presented uniformity in genotype.

Protein Extraction and Sample Preparation for 2D Electrophoresis. To reduce the biological variance and obtain an "average" sample, grains harvested from eight randomly selected maize plants for control (WT05, WT06) and transgenic (T05, T06) lines were pooled and ground with liquid nitrogen in a mortar. Afterward, protein extraction was carried out according to either Damerval et al. ${ }^{12}$ or Mechin et al. ${ }^{13}$ In the first case, an acetone solution containing $10 \%$ trichloroacetic acid and $0.07 \% \beta$-mercaptoethanol was used $(\mathrm{V}(\mathrm{mL}) / \mathrm{w}(\mathrm{g})=$ 3.33), while the second extraction procedure was based on a strongly solubilizing mixture (R2D2 buffer) containing chaotropes (5 M urea and $2 \mathrm{M}$ thiourea), detergents (2\% CHAPS and $2 \%$ SB3-10), reducing agents (20 mM DTT and $5 \mathrm{mM}$ TCEP) and $0.5 \%$ Ampholine $\mathrm{pH}$ 3-10. Moreover, direct solubilization with the typical "lysis buffer" for IEF containing $7 \mathrm{M}$ urea, $2 \mathrm{M}$ thiourea, 3\% CHAPS, 0.5\% Ampholine pH 3-10 and $5 \mathrm{mM}$ TCEP was used. With the Damerval's procedure, $0.3 \mathrm{~g}$ of flour were dissolved in $1 \mathrm{~mL}$ of extraction buffer, while with both R2D2 and lysis buffer, the buffer volume to sample weight ratio was approximately $30 \mathrm{~mL} / \mathrm{g}$. Protein extraction was performed by vortexing for $1 \mathrm{~h}$ at room temperature; after that, the mixtures were centrifuged $(20000 \mathrm{~g}$ at room temperature) and the supernatants were recovered. In the case of Damerval's method, proteins were allowed to precipitate for $1 \mathrm{~h}$ at $-20{ }^{\circ} \mathrm{C}$; after that, they were centrifuged at $20000 \mathrm{~g}$ for $15 \mathrm{~min}$ at $4{ }^{\circ} \mathrm{C}$. The pellet was washed twice with cold acetone containing $0.07 \% \beta$-mercaptoethanol, and afterward, it was solubilized either in R2D2 buffer or lysis buffer (final buffer volume to flour weight ratio was approximately $30 \mathrm{~mL} / \mathrm{g}$ ), which are compatible with the subsequent IEF analytical step. Therefore, four different protein extraction procedures were assessed: (i) Damerval's protocol + solubilization in R2D2 buffer; (ii) Damerval's protocol + solubilization in lysis buffer; (iii) direct solubilization in R2D2 buffer; and (iv) direct solubilization in lysis buffer. At the end of protein solubilization, each sample was subjected to reduction and alkylation with $5 \mathrm{mM}$ fresh TCEP and $150 \mathrm{mM}$ 2-hydroxyethyl disulfide, after which protein concentrations were determined by means of the RC-DC Protein Assay (BioRad, Hercules, CA).

2D-Electrophoresis Conditions. Four large-size maps $(18 \times$ $20 \mathrm{~cm}$ ) were prepared for both control and transgenic maize lines, using 17-cm long nonlinear IPGs $\mathrm{pH} 3-10$. Approximately 
$800 \mu \mathrm{g}$ of total proteins dissolved in $400 \mu \mathrm{L}$ was loaded on each strip by active in-gel rehydration: the strips were allowed to rehydrate for $1 \mathrm{~h}$ in the separation vessel, after which a potential of $100 \mathrm{~V}$ was applied for $7 \mathrm{~h}$. At the end of the rehydration step, the focusing process started immediately, with a linear voltage ramp up to $1000 \mathrm{~V}$ in $5 \mathrm{~h}$, followed by $5 \mathrm{~h}$ at $1000 \mathrm{~V}$ constant, a new ramp from 1000 to $10000 \mathrm{~V}$ in $1 \mathrm{~h}$, and finally $10000 \mathrm{~V}$ constant until reaching a total product of $80000 \mathrm{Vh}$. Subsequently, the strips were immediately equilibrated for $25 \mathrm{~min}$ in slow agitation with a Tris- $\mathrm{HCl}$ solution (192 mM), pH 8.8, containing 2\% SDS, $20 \%$ glycerol and $6 \mathrm{M}$ urea. The strips were then attached to the top of the seconddimension gels by means of $0.5 \%$ agarose melted in cathode buffer $(0.1 \%$ SDS in Tris-Glycine, $\mathrm{pH}$ 8.3). The second dimension was performed on Tris- $\mathrm{HCl} 8-18 \% \mathrm{~T}$ gradient polyacrylamide gels by applying the following current program: $5 \mathrm{~mA} /$ gel for $1 \mathrm{~h}, 10 \mathrm{~mA} /$ gel for $13 \mathrm{~h}$ and $20 \mathrm{~mA} /$ gel until the dye front (bromophenol blue added in the agarose solution) reached the bottom end of the gel. Finally, the proteins were revealed by Sypro Ruby staining after 1-h protein fixation in a solution made of $40 \%$ methanol, $7 \%$ acetic acid and $53 \%$ water. Destaining was performed with the same fixing solution for $1 \mathrm{~h}$ followed by $3 \mathrm{~h}$ in water. Image digitization was carried out with the Versa-Doc imaging system model 3000 (Bio-Rad, Hercules, CA).

Gel Analysis. To have statistically significant data, four replica maps were prepared for each sample. Cross-comparisons among the different samples were performed using the software PDQuest version 8.0 (Bio-Rad, Hercules, CA). Statistic analysis was performed with the Student's $t$ test (99\% confidence interval), while only 2 -fold or higher quantitative variations were taken into consideration for further analyses.

Protein Categorization. Gene Ontology (GO) lists were downloaded from TAIR Web site (http://www.arabidopsis.org/ tools/bulk/go/index.jsp): each protein was classified with respect to its cellular component, biological process, and molecular function using GO annotation. When no GO annotation was available, proteins were annotated manually based on literature searches and closely related homologues.

In-Gel Digestion. Significantly modulated (2-fold or more) protein spots were carefully cut out from Sypro Ruby stained gels and subjected to in-gel trypsin digestion according to Shevchenko and colleagues ${ }^{14}$ with minor modifications. The gel pieces were swollen in a digestion buffer containing $50 \mathrm{mM}$ $\mathrm{NH}_{4} \mathrm{HCO}_{3}$ and $12.5 \mathrm{ng} / \mu \mathrm{L}$ of trypsin (modified porcine trypsin, sequencing grade, Promega, Madison, WI) in an ice bath. After $30 \mathrm{~min}$, the supernatant was removed and discarded, $20 \mu \mathrm{L}$ of $50 \mathrm{mM} \mathrm{NH}_{4} \mathrm{HCO}_{3}$ was added to the gel pieces, and digestion was allowed to proceed at $37^{\circ} \mathrm{C}$ overnight. The supernatant containing tryptic peptides was dried by vacuum centrifugation. Prior to mass spectrometric analysis, the peptide mixtures were redissolved in $10 \mu \mathrm{L}$ of $5 \%$ FA (Formic Acid).

Protein Identification by MS/MS. Peptide mixtures were separated using a nanoflow-HPLC system (Ultimate; Switchos; Famos; LC Packings, Amsterdam, The Netherlands). A sample volume of $10 \mu \mathrm{L}$ was loaded by the autosampler onto a homemade $2 \mathrm{~cm}$ fused silica precolumn (75 $\mu \mathrm{m}$ i.d.; $375 \mu \mathrm{m}$ o.d.; Reprosil C18-AQ, $3 \mu \mathrm{m}$ (Ammerbuch-Entringen, DE)) at a flow rate of $2 \mu \mathrm{L} / \mathrm{min}$. Sequential elution of peptides was accomplished using a flow rate of $200 \mathrm{~nL} / \mathrm{min}$ and a linear gradient from solution A ( $2 \%$ acetonitrile and $0.1 \%$ formic acid) to $50 \%$ of solution B ( $98 \%$ acetonitrile and $0.1 \%$ formic acid) in 40 min over the precolumn in-line with a homemade 10-15 cm resolving column (75 $\mu$ m i.d.; $375 \mu$ m o.d.; Reprosil C18AQ, $3 \mu \mathrm{m}$ (Ammerbuch-Entringen, Germany)).

Peptides were eluted directly into a High Capacity ion Trap (model HCTplus, Bruker-Daltonik, Germany). Capillary voltage was $1.5-2 \mathrm{kV}$ and a dry gas flow rate of $10 \mathrm{~L} / \mathrm{min}$ was used with a temperature of $230{ }^{\circ} \mathrm{C}$. The scan range used was from 300 to $1800 \mathrm{~m} / \mathrm{z}$. Protein identification was performed by searching in the National Center for Biotechnology Information nonredundant database (NCBInr) using the Mascot program (http://www.matrixscience.com). The following parameters were adopted for database searches: complete carbamidomethylation of cysteines and partial oxidation of methionines, peptide mass tolerance $\pm 1.2 \mathrm{Da}$, fragment mass tolerance \pm 0.9 $\mathrm{Da}$, missed cleavages 2. For positive identification, the score of the result of $(-10 \log (P))$ had to be over the significance threshold level $(P<0.05)$.

Even though high MASCOT scores are obtained with values greater than 60 , when proteins were identified with only one peptide, a combination of automated database search and manual interpretation of peptide fragmentation spectra was used to validate protein assignments. In this manual verification, the mass error, the presence of fragment ion series, and the expected prevalence of C-terminus containing (Y-type ions) in the high mass range were all taken into account. Moreover, replicate measurements have confirmed the identity of these protein hits.

\section{Results}

In our investigation, two-dimensional gel electrophoresis (2DE) combined with mass spectrometry (MS) was used as a profiling technique in order to detect new protein products generated during nonspecific gene insertion into maize seeds by particle bombardment. One GM (event MON 810) maize seed variety 33P67, commonly used in animal feed, was subjected to comparative seed proteome analysis using the isogenic line 33P66 as reference. By Southern blotting, only one copy of the exogenous gene was found in the examined transgenic seeds (data not shown), confirming that transgenic and its relative isogenic control differed only in the presence of the transgene, as also assessed by the producer Company.

Since an important factor to be taken into account when evaluating the unintended effects of genetic modification is the natural variability existing among maize species ${ }^{15}$ and the environmental influence, we started comparing the proteomic profiles of the same variety (isogenic control) in its WT05 generation (WT05 seeds) and WT06 progenies. This enabled us to eliminate any variation related to maize variety and to determine and quantify the environmental effect on seed proteomic profiles. On the other hand, any difference detectable in the protein expression levels between WT06 and T06 seeds, originating from plants grown side-by-side in an environmentally controlled growth chamber, can be strictly related to the genome alteration as a consequence of particle bombardment. Finally, by means of two parallel comparisons between T05 versus T06 proteomes and WT05 versus WT06 proteomes, it was also possible to evaluate how transgenic plants, showing a rearranged genome as a result of the insertion of only one extra gene, responded in different ways to the same environmental conditions with respect to their isogenic controls. In Figure 1, a scheme of the workflow is shown.

For generating high-quality 2D maps, different protein extraction methods were tested: (i) direct extraction using R2D2 buffer; (ii) direct extraction using lysis buffer; (iii) the extraction 


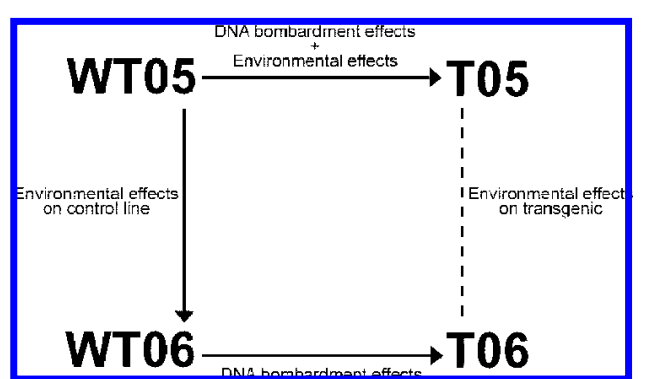

Figure 1. Experimental design carried out to discriminate the environmental influence from DNA manipulation effects on the proteomes of transgenic maize seeds and their isogenic controls when plants are grown under controlled experimental conditions. WT, wild-type; T, transgenic.

proposed by Damerval et al. ${ }^{12}$ followed by solubilization in R2D2; and (iv) the extraction described by Damerval followed by solubilization in lysis buffer. All the protein extracts obtained with the four different protocols were assayed for protein concentrations and four small-size maps were created by loading equal amounts of protein (non linear IPGs pH 3-10, 8-18\% T polyacrylamide gels). As no appreciable differences could be observed among the four procedures in terms of map quality (results not shown), direct solubilization in IEF buffer was chosen for the analysis, in the attempt to keep the extraction protocol as simple as possible and prevent possible sample loss and/or artifacts occurrence due to excessive manipulations.

Environmental Effects: Comparison of the Parental Lines (WT05 and T05) with Their Own Progenies (WT06 and T06, Respectively). We started by comparing the proteomic profiles of WT05 seeds with WT06 progenies coming from plants grown in different locations. In fact, in this event, there are no differences in the genome and the differential spots can be related to the environmental influence. This is also the case of T05 line and its progeny T06, as the two generations present the same genomic set but were grown under different environmental conditions. Figure 2 shows two representative maps of the samples WT05 and WT06, while Figure 3 displays two maps concerning the samples T05 and T06, with the differentially expressed spots highlighted with symbols and standard spot protein (SSP) numbers. Some gel regions are zoomed and displayed next to the principal images. It can be appreciated that there are both qualitative (newly induced or totally repressed spots) and quantitative (increased or decreased by at least 2-fold) changes caused by the different environments used to grow the two organisms. As concerns the couple of samples WT05/WT06, PDQuest analysis revealed that a total of 100 spots (about $30 \%$ of the total matched across all the replica 2D maps) presented significantly different expression levels in WT06 as compared to WT05. In particular, 38 spots were found to be down-regulated (i.e,, WT06 to WT05 ratio $<0.5$ ), 19 were up-regulated (ratio $>2$ ), 26 were newly expressed, while 17 were completely repressed. In the case of the transgenic samples (T05/T06), a total of 78 spots resulted to be differentially expressed, with 14 spots newly induced, 14 spots totally repressed, 23 spots up-regulated and 27 spots down-regulated in the sample T06 with respect to T05. Each of these comparisons, by itself, reveals the effects of the growth environment on the seed protein composition, as the two organisms that gave WT05 and WT06 seeds were grown in different places. But if we analyze the results of the two comparisons, we observe how two different genomes respond to the same environmental condition (WT06 and T06 were collected from plants grown in the same strictly controlled conditions), but starting from different initial circumstances chosen as references (WT05 and T05 seeds were collected from plants grown in different environments). Thus, what we observed is the combined effect of genome and environment on the final seed proteomes. This explains why the two groups of differentially expressed spots present such relevant differences (see Figures 2 and 3). Nevertheless, various protein spots result to be in common to the two groups, and in particular there are 4 commonly induced spots both in WT06 and T06 with respect to their parents (out of 26 and 14 total newly expressed spots in WT06 and T06, respectively), 3 commonly repressed spots (out of 17 and 14), 3 spots positively modulated (out of 19 and 23) and 12 spots down-regulated (out of 38 and 27). Thus, although a proper comparison between the two experiments cannot be done due to the presence of excessive variables, it is possible to conclude that the environment has a strong effect on seed protein profiles, thus leading to some common features in two organisms with different genomic sets and that start from different conditions.

Combined Effects of Genetic Manipulation and Environment: Comparison of WT05 and T05 Seed Proteomes. As WT05 and T05 seeds were collected from plants grown in slightly different environmental conditions, one should expect that possible differences in protein profiles were due to the combined action of environment and genome. However, the combination of the two factors would not necessarily lead to the arithmetic sum of the differences caused by the single factors, because each protein could be modulated either in accordance (thus resulting in a difference major than those caused by the single factors) or in opposite ways (thus possibly nullifying the single effects) by the environmental and genomic factors. In this case, in fact, only 27 protein spots resulted to be differentially expressed in T05 with respect to WT05, with 7 spots newly induced, 6 spots positively modulated, 3 spots repressed and 11 spots down-regulated. The fact that so little differences occur between these two samples with respect to the previous comparisons (100 and 78 differentially expressed protein spots in WT06 vs WT05 and T06 vs T05, respectively) might be explained by hypothesizing moderate effects of the gene insertion procedure and of the slightly different growth environments on the final seed proteome. If this was true or, to be more precise, if the growth environment had an effect on the seed proteome much greater than that of single gene insertion by particle bombardment, by comparing T06 and WT06 seeds, we should observe the effects of the sole genetic manipulation, that should affect a limited number of proteins as compared to those modulated by great environmental changes.

Effects of Single Gene Insertion on Total Protein Profile: Comparison of WT06 and T06 Seeds. To detect only unintended side effects introduced during maize gene manipulations, the two ecotypes WT05 and T05 were grown side-byside in an environmentally controlled growth chamber (but in physically separated spaces, so as to prevent cross-pollination), after which their seeds (WT06 and T06) were collected and studied. In this way, the resulting differences in proteomic profile should reflect the effects of DNA manipulation only, since the influence of variety and environment would be eliminated. 


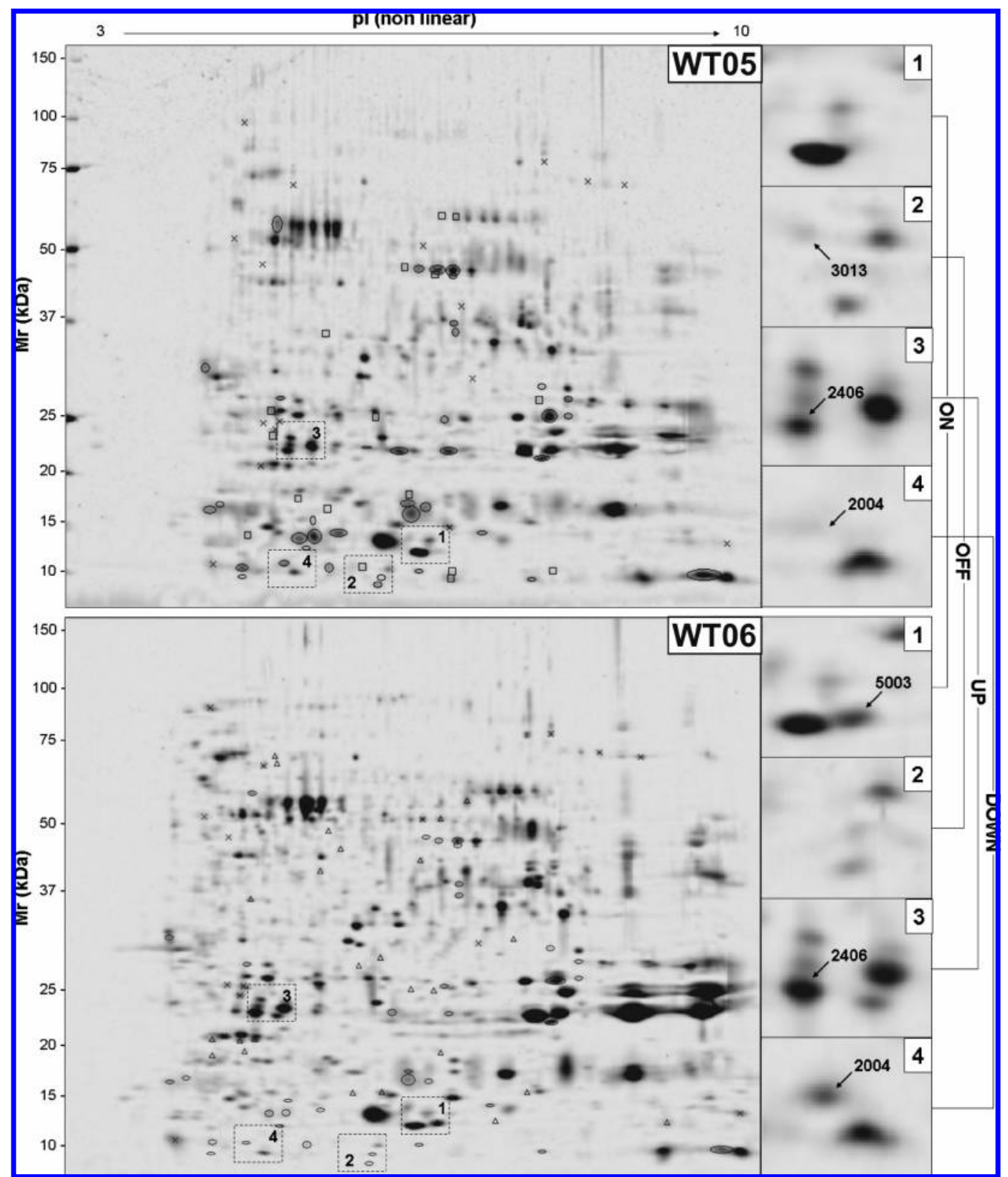

Figure 2. Comparison of WT05 and WT06 seed proteomes by 2-DE. The image displays two representative maps of WT05 and WT06 samples out of a total of 8 maps. Significantly modulated spots in WT06 as compared to WT05 are indicated by symbols: triangles, induced in WT06; squares, repressed in WT06; crosses, up-regulated in WT06; ovals, down-regulated in WT06. Dotted squares in the principal images indicate zoomed regions displayed on the right (examples of spots induced, repressed and positively or negatively modulated). Nonlinear IPGs pH 3-10 and 8-18\% T polyacrylamide gels, for the first and second dimension, respectively, were used.

Figure 4 shows the two maps concerning the samples WT06 and T06. It can be seen that there are some clear qualitative and quantitative differences, although they are much less than those observed in the comparisons concerning the environmental effects. On the other hand, the number of modulated spots is similar to that found in the previous comparison between the two parental lines. This seems to confirm the hypothesis that the environment affects protein expression more than gene manipulation by particle bombardment (in terms of total number of protein spots affected). However, it is also evident that the insertion of a single gene does not result in a unique newly expressed protein, but rather in many differently expressed genes with respect to the control. This could be due to the fact that, when the transgene enters the nucleus, many genetic loci are randomly affected by the insertion procedure. In our specific case, the transformation event resulted in a total of 43 differently expressed protein spots in T06 with respect to WT06. In particular, 14 spots were found to be down-regulated (i.e., T06 to WT06 ratio <0.5), 13 were up-regulated (ratio $>2$ ), 7 were newly expressed while 9 were completely repressed in the transgenic line. The spots of interest appear to be scattered in the entire map, with no particular restriction of molecular mass and isoelectric point. Although the particular identities of such proteins should not be of great importance in understanding or discussing the phenomenon here presented, given that changes in the expression levels should be totally accidental, nevertheless, some of the spots were cut out of the gel, digested with trypsin and 


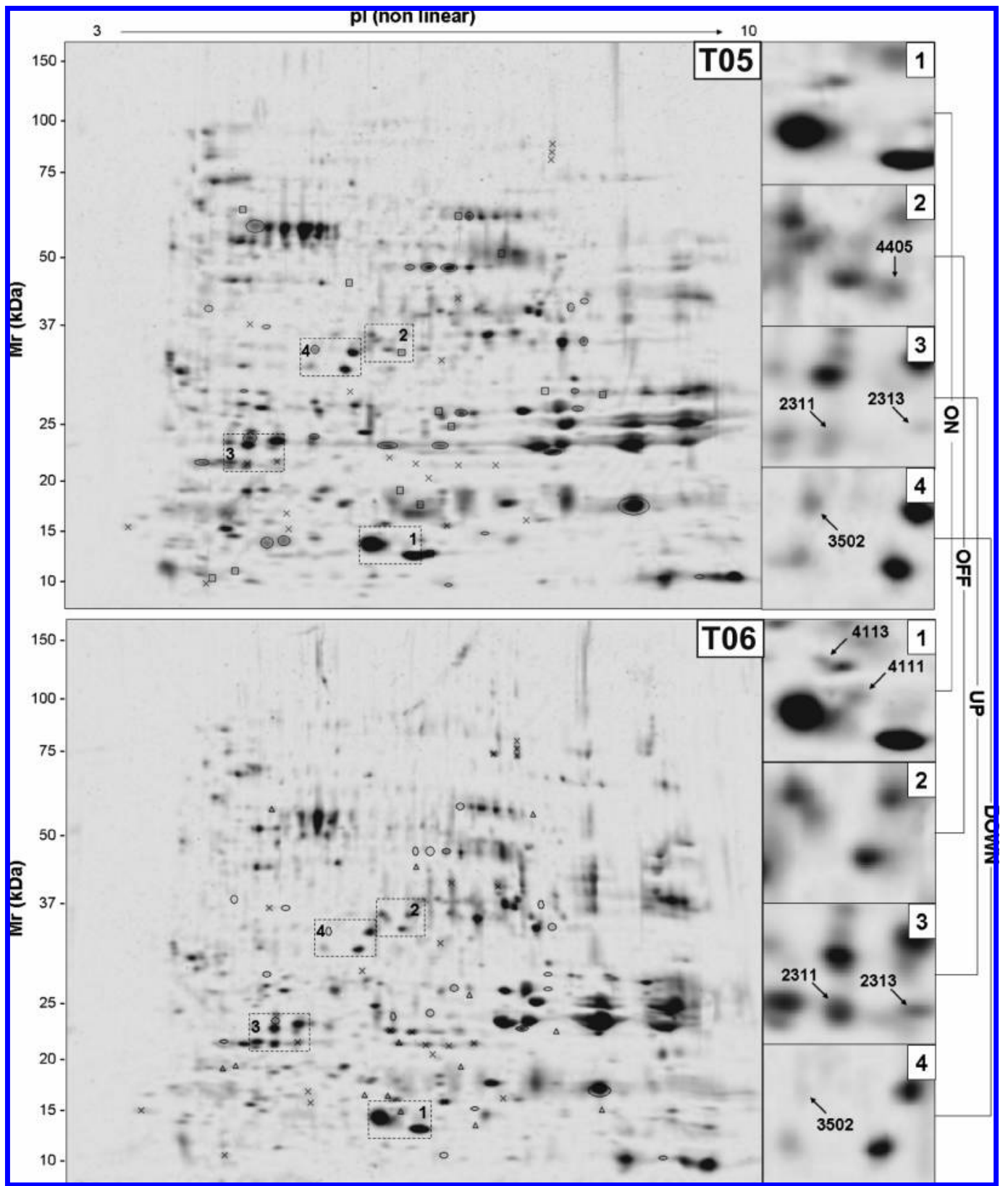

Figure 3. Comparison of T05 and T06 seed proteomes by 2-DE. The image displays two representative maps of T05 and T06 samples out of a total of 8 maps. Significantly modulated spots in T06 as compared to T05 are indicated by symbols: triangles, induced in T06; squares, repressed in T06; crosses, up-regulated in T06; ovals, down-regulated in T06. Dotted squares in the principal images indicate zoomed regions displayed on the right (examples of spots induced, repressed and positively or negatively modulated). Nonlinear IPGs $\mathrm{pH}$ 3-10 and 8-18\% T polyacrylamide gels, for the first and second dimension, respectively, were used.

analyzed by internal peptide sequencing, using mainly RPHPLC-ESI-MS/MS. In Table 1 the identities of the successfully identified proteins are listed, together with the standard spot protein number (SSP), the identification parameters, and the indication of their GO annotation (cellular component, biological process, and molecular function). Moreover, a supplemental table with peptide sequences is provided in the Supporting Information.

Interestingly, a newly expressed spot (SSP 6711) corresponding to $50 \mathrm{kDa}$ gamma zein, a well-known allergenic protein, ${ }^{16}$ has been detected. Moreover, as a major concern, a number of seed storage proteins (such as globulins and vicilin-like embryo storage proteins) exhibited truncated forms having molecular masses significantly lower than the native ones (e.g.,
SSP 7307, 2313, 8112, 1405, 2407, 5112, 2311, 5226, see Table 1). In agreement wit our data, Anderson et al., ${ }^{17}$ when analyzing various wheat lines, found that mass modifications occurred in the major storage proteins of wheat, even though less frequently than charge modifications. In our study, more vertical than horizontal position shifts were observed. A possible explanation for this observation may be that mutations leading to protein molecular mass changes are more frequent or that vertical position shifts are easier to detect than horizontal position shifts due to a higher resolution power in this direction.

Finally, neither new nor chimeric proteins have been revealed, as well as none of the protein products deriving from the marker gene inserted (Bt toxin) could be identified in our 


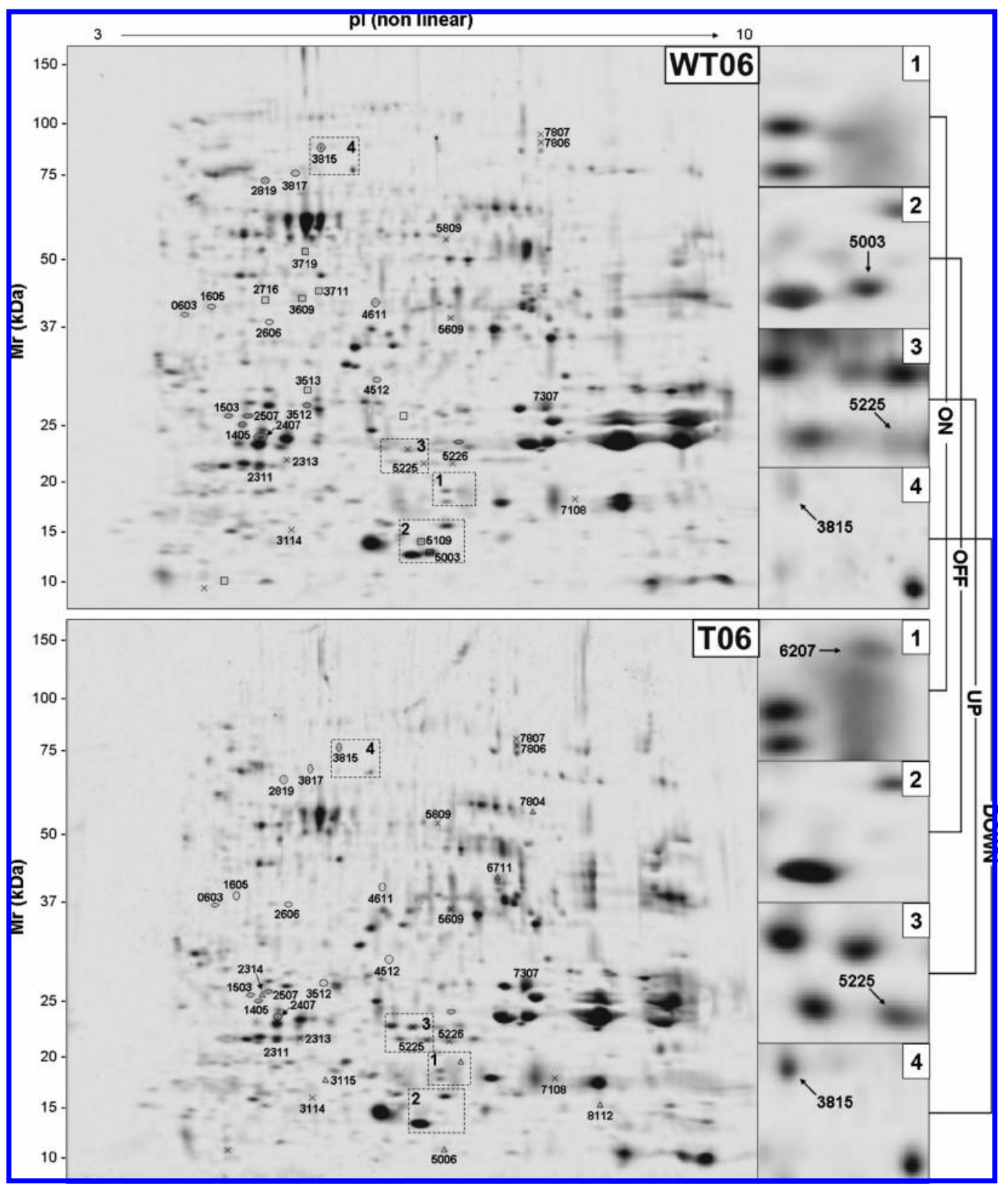

Figure 4. Comparison of WT06 and T06 seed proteomes by 2-DE. The image displays two representative maps of WT06 and T06 samples out of a total of 8 maps. Significantly modulated spots in T06 as compared to WT06 are indicated by symbols: triangles, induced in T06; squares, repressed in T06; crosses, up-regulated in T06; ovals, down-regulated in T06. Dotted squares in the principal images indicate zoomed regions displayed on the right (examples of spots induced, repressed and positively or negatively modulated). SSP numbers refer to proteins listed in Table 1. Nonlinear IPGs pH 3-10 and 8-18\% T polyacrylamide gels, for the first and second dimension, respectively, were used.

2D gels. It is presumed that the expression of the marker genes is below the limit of detection (LOD) of the method here employed. The same was observed by Corpillo and co-workers, ${ }^{18}$ who were only able to detect the product of their marker gene (nptII) by highly sensitive immuno-detection.

\section{Discussion}

The increasing presence of transgenic plant derivatives in a wide range of animal and human consumables has provoked in western Europe a strong demand for appropriate detection methods to evaluate the existence of transgenic elements. Up to now, despite the fact that confidence in the safety and reliability of crop species depends significantly on their genetic integrity, the frequency of transformation-induced mutations and their importance as potential biohazards are poorly understood. In the attempt to increase the chance of detecting unintended effects deriving from transformation events, profiling techniques such as proteomics are currently tested as analytical tools complementary to the existing safety assessment methods. Several studies have demonstrated the capacity of 2-DE of characterizing and distinguishing varieties and genotypes and even of identifying single mutations with multiple effects (for reviews, see refs 19 and 20). Proteomics has also the potential of precisely quantifying the possible allergens present in a sample, and of detecting possible posttranslational modifications. 


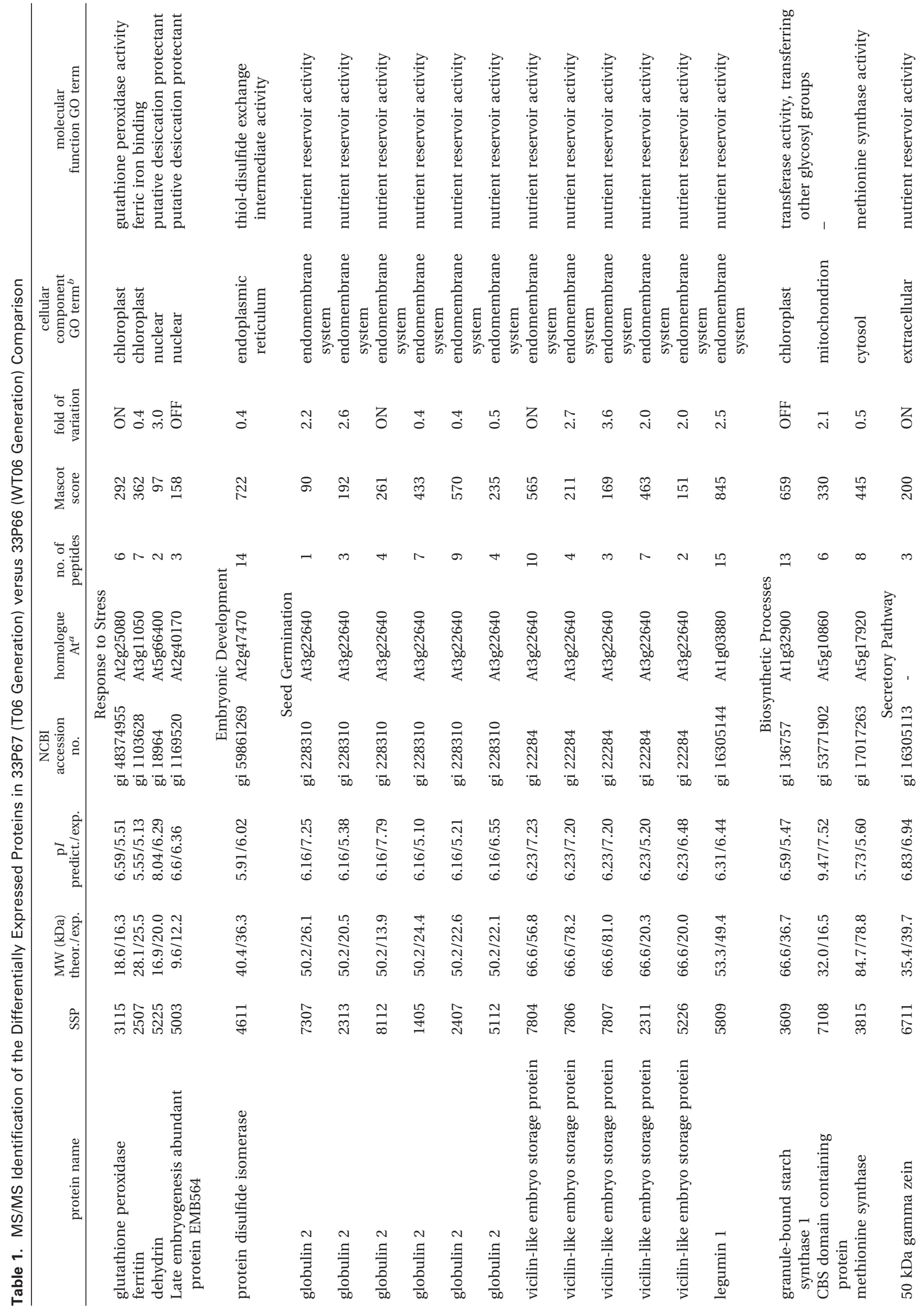




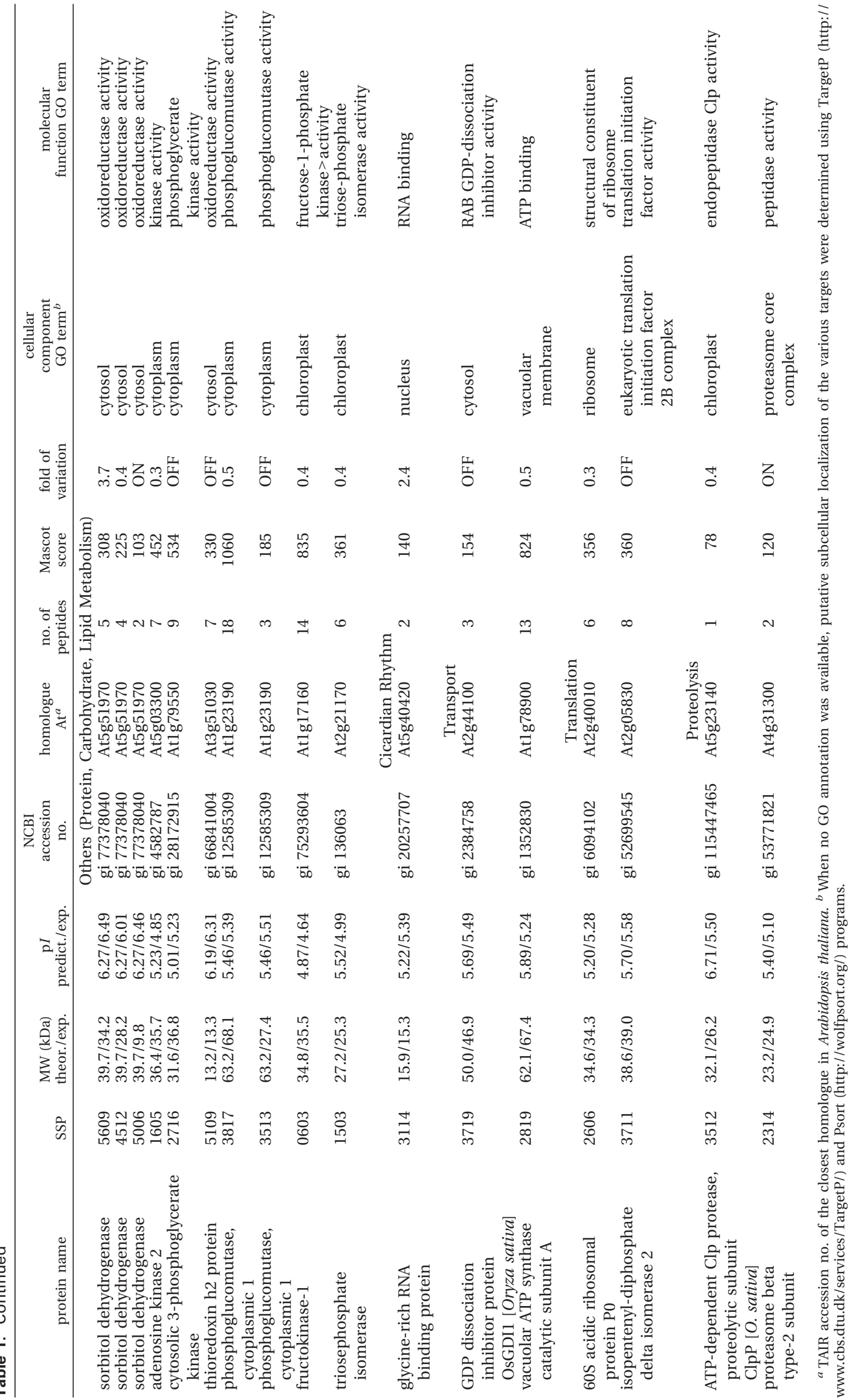


In our investigation, transgenic maize seeds (T05) and their isogenic controls (WT05) were grown side-by-side in an environmentally controlled chamber and new seeds from these plants, transgenic (T06) and relative controls (WT06), were harvested. Thus, by cross-comparing the proteomic profiles of these seeds, we were able to eliminate the natural variability existing among maize species as well as the environmental effects and, therefore, to determine in an unequivocal way the possible effects related to genome modification as a consequence of particle bombardment. Clearly the differentially regulated proteins identified in the examined transgenic seeds are specifically related to that sample, considering that particle bombardment affects the seed genome randomly, thus making accurate case by case assessments highly desirable. Data revealed that the environment affects protein expression (in terms of total number of protein spots) more than gene manipulation by particle bombardment. Moreover, transgenic plants reacted differentially to the same environmental conditions compared to their isogenic counterpart, supporting the hypothesis that they had a strongly rearranged genome after particle bombardment.

The fact that quite a high number of protein spots was found to be significantly modulated in the examined transgenic line with respect to the parental one is in contrast with the data collected by other researchers, who reported that only a few proteins had been affected by the genetic manipulation, and where the process of transformation did not seem to cause insertional or pleiotropic changes into the seed proteome. ${ }^{18,21}$ In these studies, differences in spot quantity between transgenic and nontransgenic lines fell in the range of natural variation or were part of the intended effects. They did not find any protein changes due to genetic modification either. In our opinion, the discrepancy may be attributed partly to the fact that, in those cases, genome transformation was performed by Agrobacterium instead of by particle bombardment, which is a more "destructive" technique, partly to sample preparation. The former explanation agrees with what expected: while with A. tumefaciens, the insertion sites tend to have simpler structures, but with extensive chromosomal rearrangements, with the particle bombardment approach, the variability seems to be associated with deletion and extensive scrambling of inserted and chromosomal DNA. ${ }^{22}$ The clearest evidence implicating A. tumefaciens infection as a mutagenic event comes from large-scale T-DNA tagging experiments, ${ }^{23,24}$ while it is not known yet whether particle bombardment results in chromosomal mutations, and those introduced at other random locations, commonly called genome wide mutations. In agreement with these observations, the experimental results reported by Arencibia et al. ${ }^{25}$ and by Bao et al. ${ }^{26}$ indicated that transgenic rice produced by $A$. tumefaciens treatment is characterized by fewer genomic changes than those produced with particle bombardment or cell electroporation. In this regard, so far only a handful of studies has provided detailed data on the chromosomal mutations resulting from particle bombardment insertion, ${ }^{27-30}$ but no large-scale study had been conducted on the transgene insertion patterns obtained with particle bombardment. However, it appears that transgene integration resulting from such a technique is usually accompanied by substantial disruption of the plant DNA and by the insertion of superfluous DNA (bacterial chromosomal DNA adjacent to a transgene and/or sequences close to the functional gene sequence). ${ }^{22}$ Associated rearrangements can interfere with the expression of genes containing homologous or similar sequences. ${ }^{31,32}$ A naturally occurring instance of this phenomenon has been reported for the nontransgenic rice lowglutelin-content mutation, where a deletion resulted in the transcription of a neighboring member of the glutelin gene family, but was thought to have caused the silencing of the entire gene family. ${ }^{33}$

In our investigation, a significant number of proteins were differentially regulated both by environment and by insertion of a new gene, allowing one to suppose that, when differences in spot quantity between transgenic and nontransgenic lines fell in the range of natural variation, that could be due to a poor 2D map quality or specifically related to the introduced gene. Interestingly, MS/MS identification of modulated spots revealed that the proteins involved belong to several different functional categories, but no new or chimeric proteins unique to individual GM lines were observed. Similar results have been reported for transgenic rice plants (O. sativa), where artificially induced ectopic overexpression of a single gene (YK1) in rice cells affected the expression of unrelated proteins and metabolites, ${ }^{34}$ whereas in another transgenic rice, a considerable fraction of the storage proteins turned out to be strongly underexpressed (or even completely silenced). ${ }^{35}$ As concerns these proteins, our findings demonstrated the natural presence of truncated forms although their expression levels appeared to be largely modulated after gene introduction. Since a cocktail of protease inhibitors was used during protein extraction, the phenomenon cannot be attributed to proteolytic activity, whereas it is well-known that naturally occurring gene mutations could affect the net charge (isoelectric point) and/or molecular mass of the resulting protein and, therefore, the protein pattern in two-dimensional electrophoresis (2-DE). A point mutation (base-pair substitution) of the DNA, for example, can result in (i) missense mutation, ${ }^{36}$ (ii) nonsense mutation leading to protein elongation or truncation, (iii) loss of phosphorylation or glycosylation sites, and (iv) alteration of the degradation stability of the resulting protein. Although further investigations are needed, quantitative variations here detected for the seed storage proteins may be a result of changes in the amino acid sequence (in particular, change of degradation stability) enhanced by the new gene insertion in GM seeds. On the other hand, independent analyses evidenced that previously undetected insertion-site mutations actually occurred in various commercialized products, among which is Maize YieldGard (event Mon810). ${ }^{27}$ The latter was found to contain also one truncated copy of each one of the two codelivered plasmids "interspersed with six small scrambled fragments of transgene and genomic DNA", as well as probable additional rearrangements that had not been investigated before. ${ }^{28}$ Even though the mutations found in many studies were numerous, the analytical techniques used may underestimate the true numbers because they are likely to miss most point mutations and small deletions, which instead could be revealed by proteomics.

However, it should be kept in mind that the detection of changes in protein profiles does not present a safety issue per $s e$; the relevance of such changes for food safety should be assessed (also in the context of the natural variation not investigated here) by subsequent elucidation of the nature of the proteins affected. ${ }^{37}$

\section{Concluding Remarks}

From our data it can be elicited that environment plays the main influence on proteomic profiles of transgenic seeds, but 
also that particle bombardment by itself induces additional genome alterations which cause a different protein expression. Moreover, a different response of transgenic organisms to environmental changes occurred as a consequence of a genome rearrangement in transgenic plants upon particle bombardment. In this context, it should be noted that the occurrence of unintended effects is not unique to the application of recombinant DNA techniques. It is an inherent and general phenomenon that occurs frequently in traditional breeding, for example, due to hybridization (potato breeding lines with novel, toxic glycoalkaloids ${ }^{38}$ ), natural genetic recombination, natural chromosomal rearrangements (translocations and inversions), activity of transposable elements in plant genomes, cell fusion, or chemical and radiation induced mutations. ${ }^{2,10}$ Thus it is not surprising that in vitro DNA manipulations, as performed for GM organisms, may statistically induce more unintended effects than natural recombination. However, the aim of the safety assessment of novel foods, including those produced by GM technologies, is to demonstrate that the novel food is as safe as its traditional counterpart (where one exists), thus, being totally free from any additional or new risks to consumer's health. Thus, a combination of targeted and nontargeted methods of analysis (to be chosen on a case-by-case basis) is likely to be the best way for evaluating the safety of both GM and conventionally bred crops, and for choosing the transgenic seeds having the less negative alterations. In this regard, proteomic profiling by 2-DE is a promising tool for screening purposes, and although the number of proteins that can be analyzed by 2-DE is still limited with respect to the number of proteins predicted to be present in a plant proteome, it remains the most widely used tool for high-resolution protein separation and quantification. Like other profiling methods, proteomic screening is not yet a routine practice when assessing the safety of GM products, but it has the potential of reducing uncertainty by providing many more data on crop composition than those obtained with targeted analysis alone. It could be stated that, where genomic analysis cannot reveal inadvertent modifications, proteomics could be a powerful tool. An exhaustive differential proteomic analysis allows the determination of similarities and differences between the existing food and the new products (substantial equivalence), and a case-by-case assessment of the new food should be always carried out. ${ }^{5}$ Obviously, much remains to be discovered about genome-wide and insertion-site mutations, but the lack of information, especially for crop plants and particle bombardment, means that plant transformation requires a careful investigation.

Acknowledgment. We gratefully acknowledge Dr. Giovanni Monastra (INRAN, Rome) for kindly providing the maize seeds. This work was financially supported by grants from the Italian Ministry for University Research (MIURPRIN 2006) and Progetto MIPAF Genzoot 2006.

Supporting Information Available: Table of peptide sequences obtained by MS/MS experiments from T06 versus WT06 comparison. This material is available free of charge via the Internet at http://pubs.acs.org.

\section{References}

(1) Somers, D. A.; Makarevitch, I. Transgene integration in plants: poking or patching holes in promiscuous genomes. Curr. Opin. Biotechnol. 2004, 15, 126-131.

(2) Conner, A. J.; Jacobs, J. M. Genetic engineering of crops as potential source of genetic hazard in the human diet. Mutat. Res. 1999, 443, 223-234.
(3) Ruebelt, M. C.; Lipp, M.; Reynolds, T.; Schmuke, J. J.; Astwood, J. D.; DellaPenna, D.; Engel, K. H.; Jany, K. D. Application of twodimensional gel electrophoresis interrogate alterations in the proteome of genetically crops. 3 . Assessing unintended effects. $\underline{L}$ Agric. Food Chem. 2006, 54, 2169-2177.

(4) Cellini, F.; Chesson, A.; Colquhoun, I.; Constable, A.; Davies, H. V.; Engel, K. H.; Gatehouse, A. M. R.; Karenlampi, S.; Kok, E. J.; Leguay, J.-J.; Lehesranta, S.; Noteborn, H. P.; Pedersen, J.; Smith, M. Unintended effects and their detection in genetically modified crops. Food Chem. Toxicol. 2004, 42, 1089-1125.

(5) Kuiper, H. A.; Kleter, G. A.; Noteborn, H.P.J.M.; Kok, E. J. Assessment of the food safety issues related to genetically modified foods. Plant J. 2001, 27, 503-528.

(6) GM Science Review Second Report, an open review of the science relevant to GM crops and food based on interests and concerns of the public. 2004, $116 \mathrm{pp}$, http://www.gmsciencedebate.org.uk/ report/.

(7) Society of Toxicology. The safety of genetically modified foods produced through biotechnology. Toxicol. Sci. 2003, 71, 2-8.

(8) Chassy, B.; Hlywka, J. J.; Kleter, G. A.; Kok, E. J.; Kuiper, H. A.; McGloughlin, M.; Munro, I. C.; Phipps, R. H.; Reid, J. E. Nutritional and safety assessments of foods and feeds nutritionally improved through biotechnology: an executive summary. Compr. Rev. Food Sci. Food Saf. 2004, 3, 38-104.

(9) Wilkins, M. R.; Williams, K. L.; Appel, R. D.; Hochstrasser, D. F. Proteome Research: New Frontiers in Functional Genomics; Springer: Berlin, 1997.

(10) FAO/WHO. Report of the Third Session Of the Codex ad hoc Intergovernmental Task Force on Foods Derived from Biotechnology, Yokohama, Japan; Codex Alimentarius Commission, FAO: Rome, 2002.

(11) Hamdan, M.; Righetti, P. G. Proteomics Today: Protein Assessment and Biomarkers Using Mass Spectrometry, 2-D Electrophoresis and Microarray Technology; Wiley-VCH: Hoboken, NJ, 2005.

(12) Damerval, C.; De Vienne, D.; Zivy, M.; Thiellement, H. Technical improvements in two-dimensional electrophoresis increase the level of genetic variation detected in wheat-seedling proteins. Electrophoresis 1986, 7, 52-54.

(13) Mechin, V.; Consoli, L.; Le Guilloux, M.; Damerval, C. An efficient solubilization buffer for plant proteins focused in immobilized $\mathrm{pH}$ gradients. Proteomics 2003, 3, 1299-1302.

(14) Shevchenko, A.; Wilm, M.; Vorm, O.; Mann, M. Mass spectrometric sequencing of proteins silver-stained polyacrylamide gels. Anal. Chem. 1996, 68, 850-858.

(15) Consoli, L.; Damerval, C. Quantification of individual zein isoforms resolved by two-dimensional electrophoresis: genetic variability in 45 maize inbred lines. Electrophoresis 2001, 22, 2983-2989.

(16) Pasini, G.; Simonato, B.; Curioni, A.; Vincenzi, S.; Cristaudo, A.; Santucci, B.; Peruffo, A. D.; Giannattasio, M. IgE-mediated allergy to corn: a $50 \mathrm{kDa}$ protein, belonging to the Reduced Solubile Proteins, is a major allergen. Allergy 2002, 57, 98-106.

(17) Anderson, N. G.; Tollaksen, S. L.; Pascoe, F. H.; Anderson, L. Twodimensional electrophoretic analysis of wheat seed proteins. Crop Sci. 1985, 25, 667-674.

(18) Corpillo, D.; Gardini, G.; Vaira, A. M.; Basso, M.; Aime, S.; Accotto, G. P.; Fasano, M. Proteomics as a tool to improve investigation of substantial equivalence in genetically modified organisms: the case of a virus-resistant tomato. Proteomics 2004, 4, 193-200.

(19) de Vienne, D.; Bost, B.; Fievet, J.; Zivy, M.; Dillmann, C. Genetic variability of proteome expression and metabolic control. Plant Physiol. Biochem. 2001, 39, 271-283.

(20) Canovas, F. M.; Dumas-Gaudot, E.; Recorbet, G.; Jorrin, J.; Mock, H. P.; Rossignol, M. Plant proteome analysis. Proteomics 2004, 4, 285-298.

(21) Lehesranta, S. J.; Davies, H. V.; Shepherd, L. V.; Nunan, N.; McNicol, J. W.; Auriola, S.; Koistinen, K. M.; Suomalainen, S.; Kokko, H. I.; Karenlampi, S. O. Comparison of tuber proteomes of potato varieties, landraces, and genetically modified lines. $\underline{\text { Plant }}$ Phvsiol. 2005, 138, 1690-1699.

(22) Latham, J. R.; Wilson, A. K.; Steinbrecher, R. A. The Mutational Consequences of Plant Transformation. $\underline{\text { J.Biomed. Biotechnol. }}$. 2006, 25376, 1-7.

(23) Budziszewski, G. J.; Lewis, S. P.; Glover, L. W.; Reineke, J.; Jones, G.; Ziemnik, L. S.; Lonowski, J.; Nyfeler, B.; Aux, G.; Zhou, Q.; McElver, J.; Patton, D. A.; Martienssen, R.; Grossniklaus, U.; Ma, H.; Law, M.; Levin, J. Z. Arabidopsis genes essential for seedling viability: isolation of insertional mutants and molecular cloning. Genetics 2001, 159, 1765-1778.

(24) Castle, L. A.; Errampalli, D.; Atherton, T. L.; Franzmann, L. H.; Yoon, E. S.; Meinke, D. W. Genetic and molecular characterization 
of embryonic mutants identified following seed transformation in Arabidopsis. Mol. Gen. Genetics 1993, 241, 504-514.

(25) Arencibia, A.; Gentinetta, E.; Cuzzoni, E.; Castiglione, S.; Kohli, A.; Vain, P.; Leech, M.; Christou, P.; Sala, F. Molecular analysis of the genome of transgenic rice (Oryza sativa L.) plants produced via particle bombardment or intact cell electroporation. Mol. Breeding 1998, 4, 99-109.

(26) Bao, P. H.; Granata, S.; Castiglione, S.; Wang, G.; Giordani, C.; Cuzzoni, E.; Damiani, G.; Bandi, C.; Datta, S. K.; Datta, K.; Potrykus, I.; Callegarin, A.; Sala, F. Evidence for genomic changes in transgenic rice (Oryza sativa L.) recovered from protoplasts. Transgenic Res. 1996, 5, 97-103.

(27) Hernandez, M.; Pla, M.; Esteve, T.; Prat, S.; Puigdomenech, P.; Ferrando, A. A specific real-time quantitative PCR detection system for event MON810 in maize YieldGard based on the 3'-transgene integration sequence. Transgenic Res. 2003, 12, 179-189.

(28) Makarevitch, I.; Svitashev, S. K.; Somers, D. A. Complete sequence analysis of transgene loci from plants transformed via microprojectile bombardment. Plant Mol. Biol. 2003, 52, 421-432.

(29) Shimizu, K.; Takahashi, M.; Goshima, N.; Kawakami, S.; Irifune, K.; Morikawa, H. Presence of an SAR-like sequence in junction regions between an introduced transgene and genomic DNA of cultured tobacco cells: its effect on transformation frequency. $\underline{\text { Plant }}$ J. 2001, 26, 375-384.

(30) Windels, P.; Taverniers, I.; Depicker, A.; Van Bockstaele, E.; De Loose, M. Characterisation of the Roundup Ready soybean insert. Eur. Food Res.Technol. 2001, 213, 107-112.

(31) Hannon, G. J. RNA interference. Nature 2002, 418, 244-251.
(32) Bartel, B.; Bartel, D. P. MicroRNAs: at the root of plant development. Plant Physiol. 2003, 132, 709-717.

(33) Kusaba, M.; Miyahara, K.; Iida, S.; Fukuoka, H.; Takano, T.; Sassa, H.; Nishimura, M.; Nishio, T. Low glutelin content1: a dominant mutation that suppresses the glutelin multigene family via RNA silencing in rice. Plant Cell. 2003, 15, 1455-1467.

(34) Takahashi, H.; Hotta, Y.; Hayashi, M.; Kawai-Yamada, M.; Komatsu, S.; Uchimiya, H. High throughput metabolome and proteome analysis of transgenic rice plants (Oryza sativa L.). Plant Biotechnol. 2005, 22, 47-50.

(35) Islam, N.; Upadhyaya, N. M.; Campbell, P. M.; Akhurst, R.; Hagan, N.; Higgins, T. J. V. Decreased accumulation of glutelin types in rice grains constitutively expressing a sunflower seed albumin gene. Phytochemistry 2005, 66, 2534-2539.

(36) Koornneef, M.; Alonso-Blanco, C.; Vreugdenhil, D. Naturally occurring genetic variation in Arabidopsis thaliana. Annu. Rev. Plant Biol. 2004, 55, 141-172.

(37) Ruebelt, M. C.; Lipp, M.; Reynolds, T. L.; Astwood, J. D.; Engel, K. H.; Jany, K. D. Application of two-dimensional gel electrophoresis to interrogate alterations in the proteome of genetically modified crops. 2. Assessing natural variability. L. Agric. Food Chem. 2006, 54, 2162-2168.

(38) van Gelder, W. M. J.; Scheffer, J. J. C. Transmission of steroidal glycoalkaloids from Solanum Vernei to the cultivated potato. Phytochemistry 1991, 30, 165-168.

PR0705082 